\title{
Orthophotoplan Segmentation and Colorimetric Invariants for Roof Detection
}

\author{
Youssef El Merabet ${ }^{1,2}$, Cyril Meurie ${ }^{1}$, Yassine Ruichek ${ }^{1}$, Abderrahmane Sbihi ${ }^{3}$, \\ and Rajaa Touahni ${ }^{2}$ \\ ${ }^{1}$ Systems and Transportation Laboratory, Université de Technologie de \\ Belfort-Montbliard, 13 rue Ernest Thierry-Mieg, 90010 Belfort, France \\ ${ }^{2}$ Laboratoire LASTID, Département de Physique, Faculté des Sciences, \\ Université Ibn Tofail, B.P 133, 14000 Kénitra, Maroc \\ ${ }^{3}$ ENSA, Université Abdelmalek Essadi, Route Ziaten, km 10, BP 1818, \\ Tanger Maroc \\ \{youssef.el-merabet, cyril.meurie, yassine.ruichek\}@utbm.fr, \\ sbihi_abderrahmane@yahoo.fr, rtouahni@hotmail.com
}

\begin{abstract}
In this paper, we use a morphological segmentation method called watershed for segmenting roof of "orthophotoplan" images. This work takes place in a global approach which consists in recognizing a roof of aerial images among a knowledge database and bending out 3D models automatically generated from geographical data. The main aim of this work consists in defining the best couple of colorimetric invariant/gradient (among 24 colorimetric invariants and 14 gradients tested) used as input of watershed algorithm in order to obtain the best segmentation of roof. The tests are made on a database of 67 roofs containing a certain heterogeneity (illumination changes, shadows, etc) and evaluated with the Vinet criteria (including a ground truth image) in order to prove the robustness of the proposed strategy.
\end{abstract}

Keywords: watershed, color gradient, colorimetric invariant, orthophotoplan.

\section{Introduction}

The works presented in this paper appear in a global approach that consists in recognizing a roof of aerial images among a knowledge database and bending out 3D models automatically generated from geographical data. The first step of the global approach presented in this paper consists in segmenting the roof in different regions of interest in order to provide several measures of the roof (section of roof, chimneys, roof light, etc). To do that, we use a morphological segmentation method called watershed. But this method requires two input images (seed and potential images) that we have optimized for the application. A first difficulties consists in using an appropriate potential image (gradient image) in order to extract as well as possible the details of the roof (chimneys, roof light, etc). For that, several tests have been performed to define the best suitable 
gradient among a set of 14 gradients ( 8 gray levels gradients and 6 color gradients). A second difficulties concerns the recognition of the roof among a database including the same roof with different illumination changes and shadows. That is why, we propose in this paper to choose an appropriate colorimetric invariant among a set of 24 invariants extracted of the literature to limit these effects. All tests presented in this paper have been performed on an "orthophotoplan" image containing 67 roofs. Each roof to segment is extracted of the "orthophotoplan" image from the ground track (red border in Figure 1). Figure 11 illustrates an "orthophotoplan" image (left), a zoom of one roof to extract/recognize (middle) and the segmented image to obtain (right). The paper is organized as follows: Section 2 details the proposed approach including a recall of different invariants and the watershed algorithm. In section 3, we detail all tests which are permit to choose the optimal couple of invariant/gradient of the watershed algorithm for the given application. Finally we conclude and present perspective of future works.
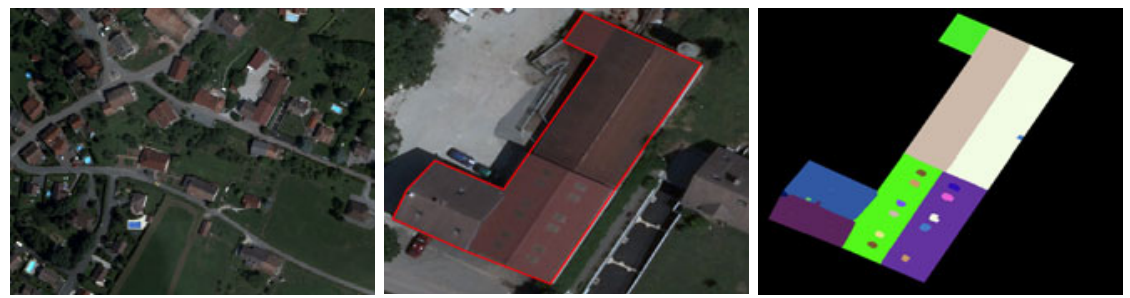

Fig. 1. Example of "orthophotoplan" image (left to right: an "orthophotoplan" image, a zoom of roof, the segmented image)

\section{Image Segmentation}

Image segmentation consists in partitioning an image in more or less regular or homogeneous regions according to a given criteria. Many segmentation methods exist in the literature. These one can be grouped in three categories: $1 /$ region based segmentation (split and merge, region growing in which we find the watershed algorithm used in this paper); 2 / edge based segmentation; 3 / classification, clustering, thresholding.

In this paper, we use the first method which corresponds to the region based segmentation and particulary a morphological method called color watershed that offers in general very good results. Thus, we will define the best couple invariant/gradient used as input images of the watershed algorithm.

Figure 2 illustrates the synopsis of the global proposed approach. It is composed of several steps. The first one corresponds to a colorimetric invariant to apply on the initial image. After that, we calculate a color gradient or a gray level gradient on the simplified image. In the case of the gray level gradient, we must extract the three component of the simplified image and calculate the 


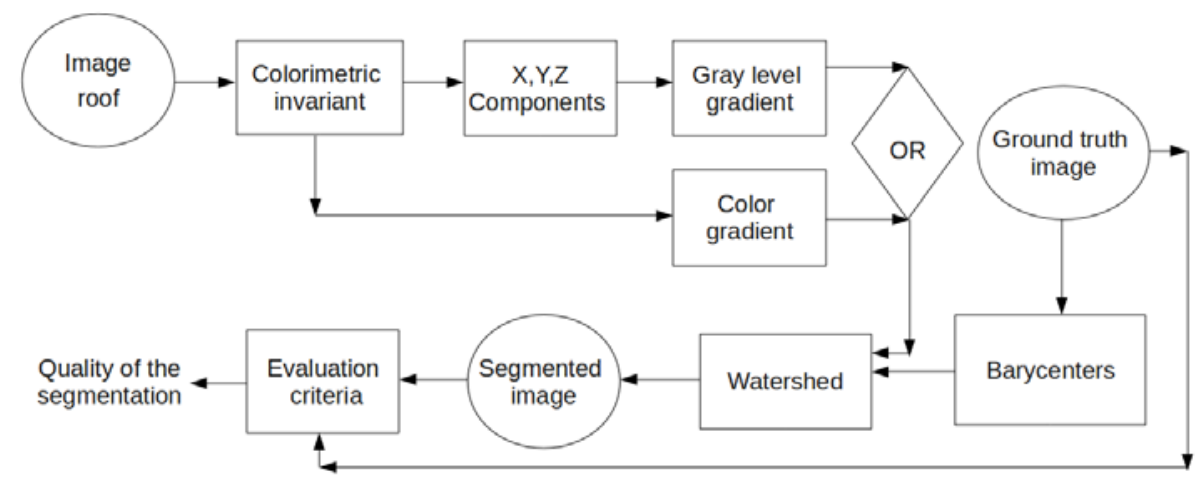

Fig. 2. Synopsis of the proposed approach

gradient on these component. The next step consists in using the watershed with the gradient image and a seed image (where a seed corresponds to the barycenter of each region of the ground truth image). Finally, we obtain the segmented image and evaluate the quality of the segmentation, with the Vinet criteria (and the reference segmentation).

\subsection{Colorimetric Invariants}

In our application, the "orthophotoplan" images contain a certain heterogeneity in terms of lights, illumination changes, shadows, etc. It does not permit to extract correctly the different regions of interest of the roof. To overcome these drawbacks, we rejoin the strategy of many authors by simplifying the input image with a suitable colorimetric invariant([1, 2], 3], 4], 5]).

Indeed, for few years, the color invariance generate much interest and continues to engage the field of computer vision. For example, one can cite the use of colorimetric invariant for matching images [4, for motion estimation in video sequences [5], for feature extraction and re-identification of individuals in transport environment 2], for enhancing the monitoring of points of interest in color images [3], etc.

In this paper, we want to show that using a colorimetric invariant can limit artefacts of the acquired image and thus obtain a better segmentation of the roof. That is why, we propose to define the best colorimetric invariant according to the proposed approach. 24 colorimetric invariants of the literature and listed below have been tested: Greyworld normalization (called Greyworld in Figures 4, 6) [6], RGB-rang [7, affine normalization (called affine in Figures 4, 6) [6], intensity normalization (called chromaticity in Figures 4, 6) [10], comprehensive color normalization (called comprehensive in Figures 4, 6] 6], c1c2c3 ([4, [1]), m1m2m3 [4, 111213 (4], 1]), 141516 [11], A1A2A3 [3], c4c5c6 [11], hsl, MaxRGB [10], Cr$\mathrm{CgCb}$ 3, Color Constant Color Indexing (called CCCI in Figures 4, 6] [10], m4m5m6 3], Standard L2 (called L2 in Figures 4, 6) 3], Maximum-intensity normalization (called Mintensity in Figures 4, 6) [12], reduced coordinates 9], 


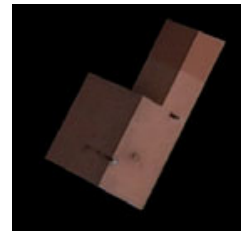

a

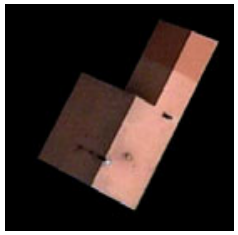

$\mathrm{b}$

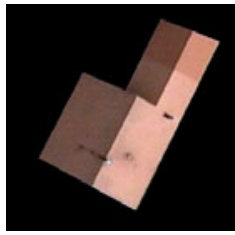

C

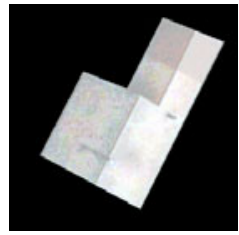

d

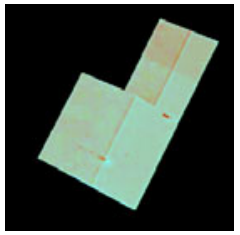

e

Fig. 3. Example of colorimetric invariants, (a) without colorimetric invariant, (b) with affine normalization, (c) with Maximum-intensity normalization, (d) with RGB-rang, (e) with c1c2c3

$\mathrm{CrCb}([3],[1])$, opposite colors (o1o2) ([3], [1]), Saturation S [4], Log-Hue [10] and Hue $\mathrm{H}([4,9])$.

Figure 3 illustrates the influence of 4 colorimetric invariants applied on the initial image.

\subsection{Watershed Algorithm}

As indicated previously, we have chosen to use a morphological segmentation method called watershed which offers generally good result. Many versions of this algorithm exist in the literature ([13], [14] [15]), but we have chosen the version presented by Meyer ([14], [16]) that we recalled below :

This algorithm extends as soon as possible, the local minima of the image (in our case: the seed calculated on the reference image) using the priority given by a potential or gradient image. It is composed of 4 steps (algorithm 1).

The watershed algorithm requires two input images (a seed and a gradient/potential images), that we detail below:

The gradient or potential image: In order to define the best gradient for our application, eight gray level gradients and six color gradients have been tested. The eight gray level gradients are: 1/Sobel; 2/Roberts; 3/Prewitt; 4/GradientF (the first derivative of the image); 5/NonMaximaSuppression (the non maxima values from the magnitude of the gradient); 6/Shen; 7/Deriche; 8/GradientM (morphological gradient corresponding to the substraction between dilated image and eroded image). The six color gradients are: 1/Di-Zenzo [17]; 2/GradientMC (morphological gradient corresponding to the substraction between dilation and erosion using a lexicographical order); 3/GradientC (the marginal gradient) [18]; 4/SobelC (Sobel calculated on color image); 5/SobelTLS (Sobel calculated in TSL color space); 6/Carron [19].

The seed image: In the watershed algorithm, the seed image corresponds generally to the local minima of the gradient image. But, it leads to an oversegmentation of the image. To overcome this drawback, a solution consists to use a selection of local minima such as defined in [20] and which offers generally good results. But in order to define in the best conditions, the couple of invariant/gradient which offers the best segmentation results, we have chosen to use 
the knowledge of the application. In fact, we calculate the barycenter of each region of the ground truth image and consider that it correspond to a seed.

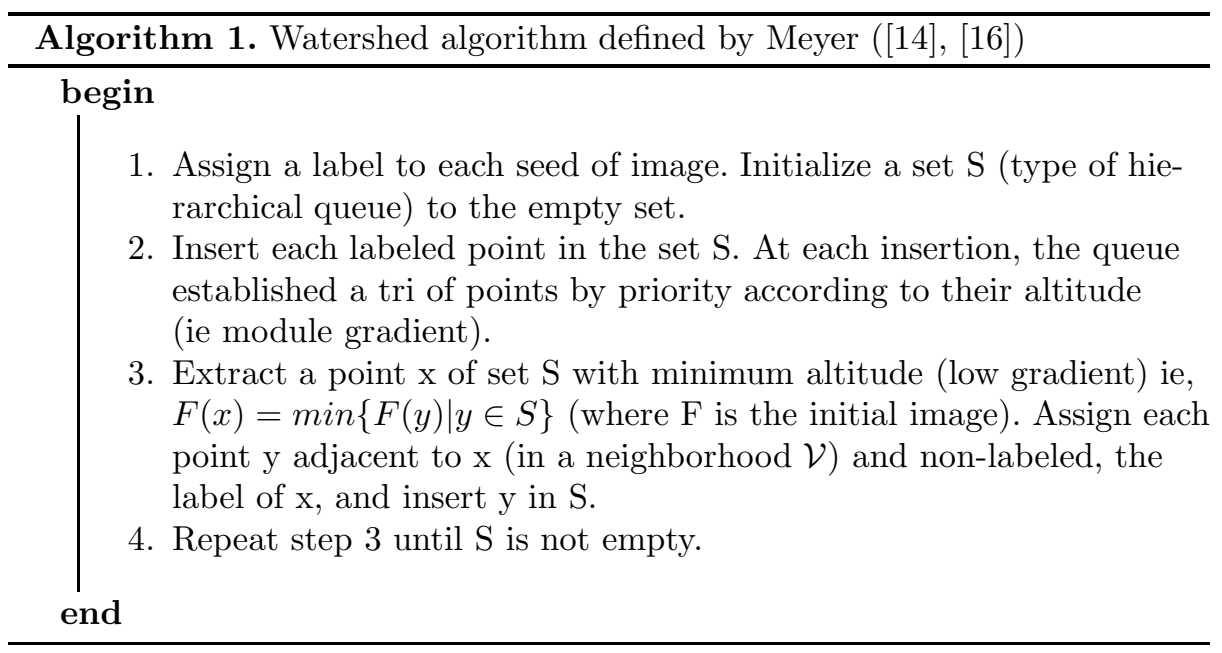

\section{$3 \quad$ Experimental Results}

To compare the segmentation results obtained with different gradients and colorimetric invariants, an appropriate evaluation is then necessary. Many evaluation methods exist in the literature. These can be classified into two categories: without and with ground truth (reference segmentation). In our application, we have used the Vinet criteria [21] which belongs to the second category. The evaluation of the segmentation results presented in this paper has been made on one "orthophotoplan" image containing 67 roofs. Each roof of the "orthophotoplan" image is associated to a ground truth (reference segmentation) created manually by a human expert. For a better readability, we have only presented eighteen better invariants among the 24 tested. As indicated in the section 2, the proposed approach uses the watershed algorithm (with an appropriate seed and potential images) on a simplified image (with a colorimetric invariant).

To define the best couple colorimetric invariant/gradient, we separate all tests in two categories. The first one corresponds to the best couple color gradient/colorimetric invariant applied directly on the simplified image (cf Figure 6). The second one corresponds to the best couple gray level gradient/component of the colorimetric invariant applied on the component of the simplified image (cf Figure 4). In this last case, $\mathrm{X}, \mathrm{Y}$ and $\mathrm{Z}$ represents respectively the first, second and third component of the colorimetric invariant. For example, hsl-Y corresponds to the second component: the saturation $(\mathrm{S})$.

Figures 4 and 6] illustrate respectively the best couple component (of the colorimetric invariant)/gray level gradient and the best couple colorimetric invariant/color gradient. For both figures, each bar represents the mean value of Vinet calculated on all images of database according to the couple invariant/gradient 

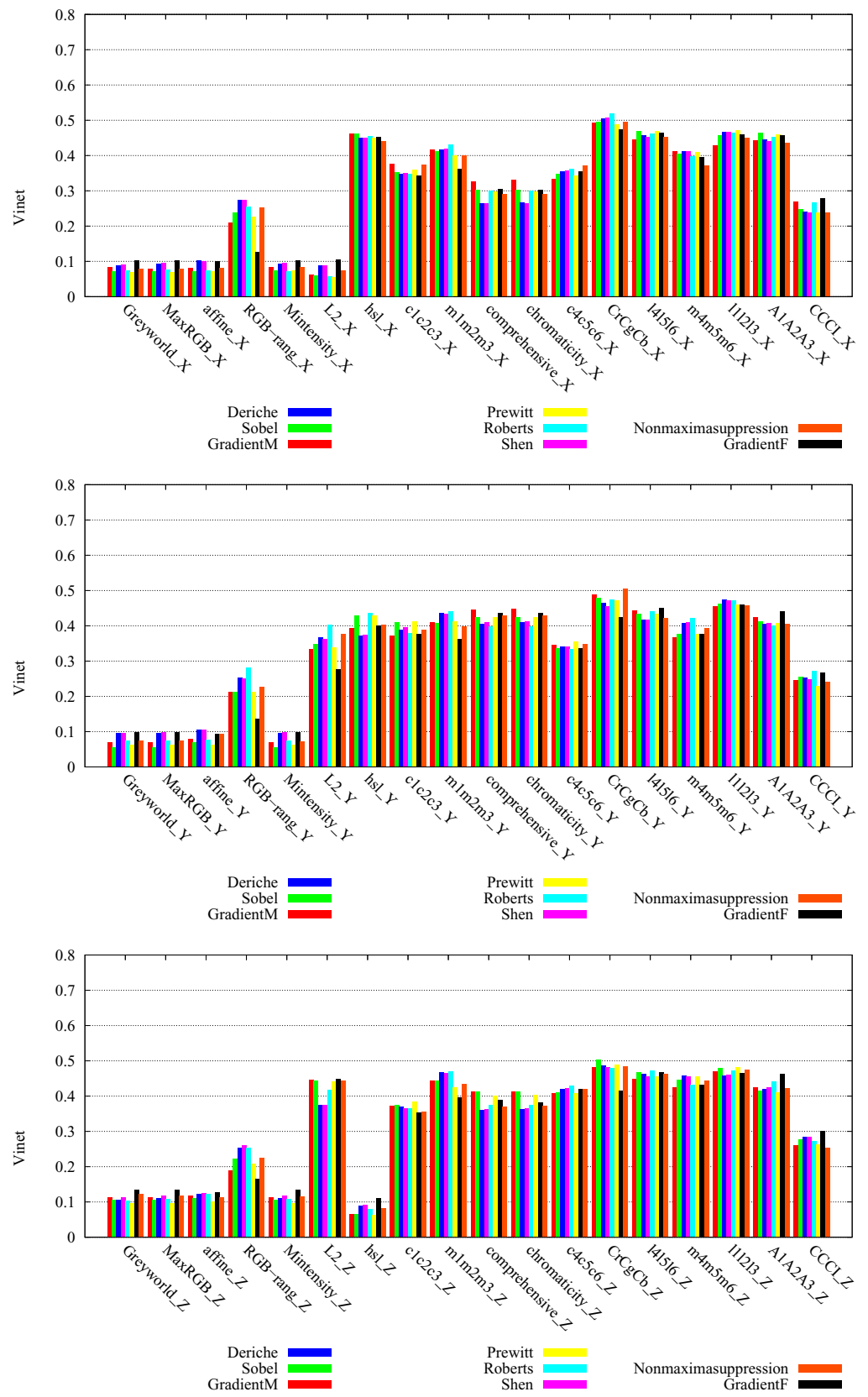

Fig. 4. Quality of the segmentation on gray level image (using Vinet criteria) according to the couple colorimetric invariant/gradient. (top to bottom: segmentation results on the X component (top), Y component (middle), Z component (bottom) of the color image). 

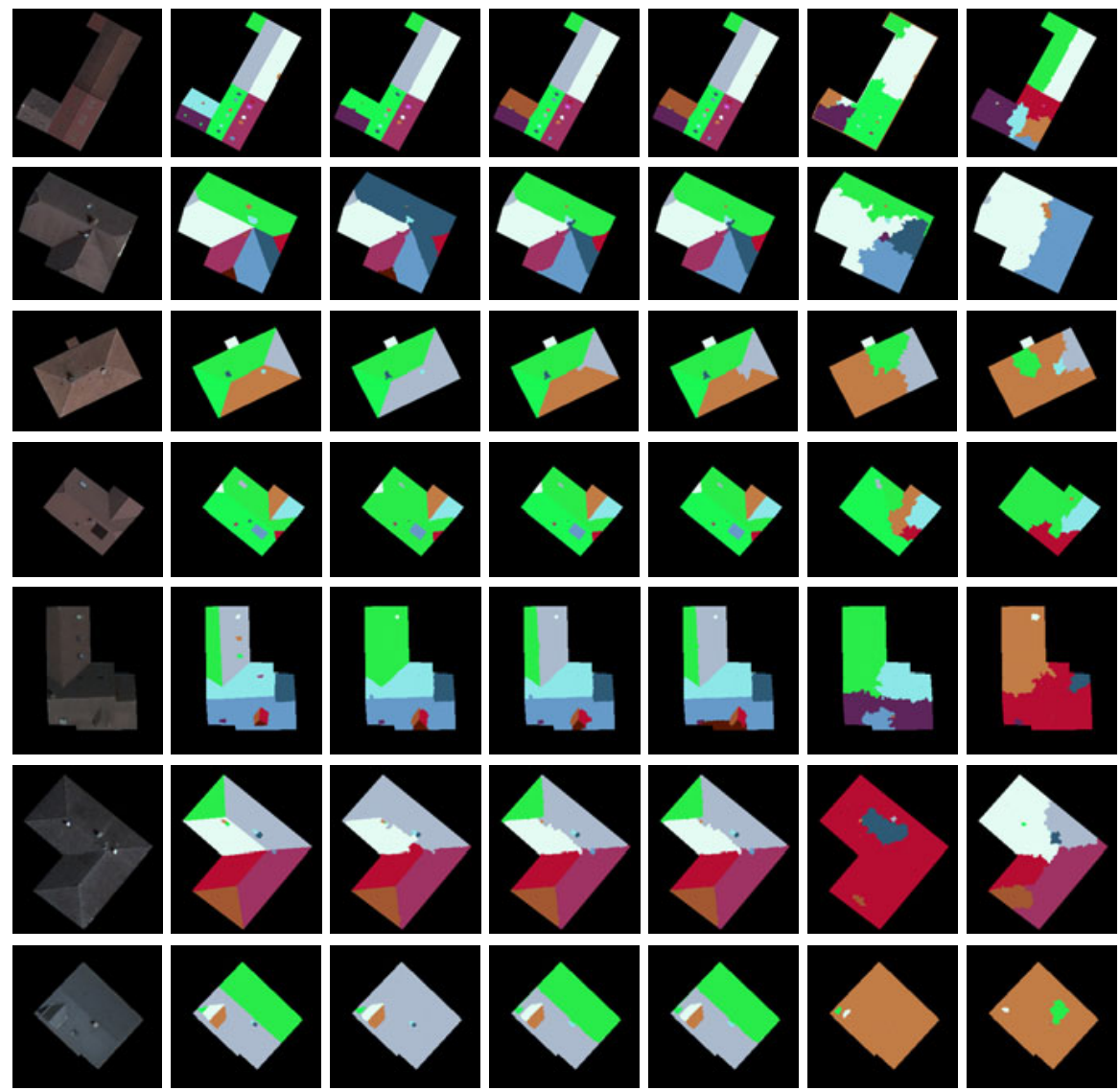

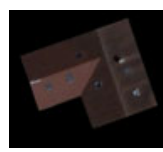

a

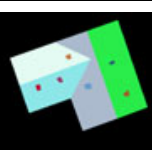

$\mathrm{b}$

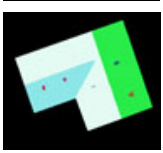

C

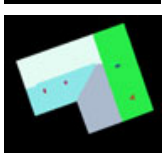

d

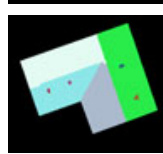

e

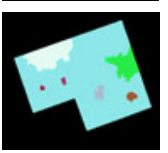

f

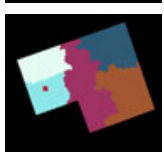

g

Fig. 5. Illustration of few segmented images obtained without/with colorimetric invariant. Initial image (a), ground truth image (b), segmented image without invariant (c), with Maximum-intensity normalization (d), with Max-RGB (e), with 111213 (f) and with $\mathrm{m} 1 \mathrm{~m} 2 \mathrm{~m} 3(\mathrm{~g})$.

tested (the results are better if the value is low). For example, considering the first component of Greyworld invariant (Greyworld-X), the first bar (in red color) corresponds to the Vinet value calculated with the GradientM gradient.

Considering the gradient calculated of the gray level image (cf Figure 4), the segmentation results depends highly of the color component selected. Indeed, for the three X, Y and Z components of the colorimetric invariants Greyworld, affine normalization, RGB-rang, Maximum-intensity normalization, and MaxRGB 
give satisfying results (the values of Vinet are low compared to those obtained with the components of the other colorimetric invariants) and whatever the gradient used. But, if we select only the best component, one can notice that the $\mathrm{X}$ component of the L2 normalization (L2-X) and the Z component of the hsl space (hsl-Z) give the best segmentation results. Finally, we can define a preference order to use with a couple of gray level gradient/component of colorimetric invariant: Prewitt/L2-X, Roberts/L2-X, GradientM/L2-X, Deriche/Mintensity-Y.

Considering the gradient calculated on the color image (cf Figure 6), Greyworld, affine normalization,RGB-rang, Maximum-intensity normalization and MaxRGB, give the best segmentation results, whatever the color gradient used even if the couple Di-Zenzo/Mintensity is the best. We can also define a preference order to use with a couple of color gradient/colorimetric invariant: DiZenzo/Mintensity, Di-Zenzo/MaxRGB, Di-Zenzo/Greyworld, Di-Zenzo/affine normalization, color morphological gradient/MaxRGB, GradientC/Mintensity. For a better visualization, the figure 5 illustrates few segmentation results obtained by the proposed approach. The Di-Zenzo gradient is used for this illustration since it permits to obtain the best results. One can notice that segmentations using an appropriate gradient/invariant offers better results than those obtained without colorimetric invariant. The segmentation results obtained with Maximum-intensity normalization and MaxRGB and presented on the figure 5 are satisfying and confirm the conclusion given about the figure 6 . The segmentations obtained without and with 111213 (cf Figure 5(f)), m1m2m3 (cf Figure 5(g)) colorimetric invariants appear weak. It is illustrating by a partial or total loss of roof information with worse quality of edge.

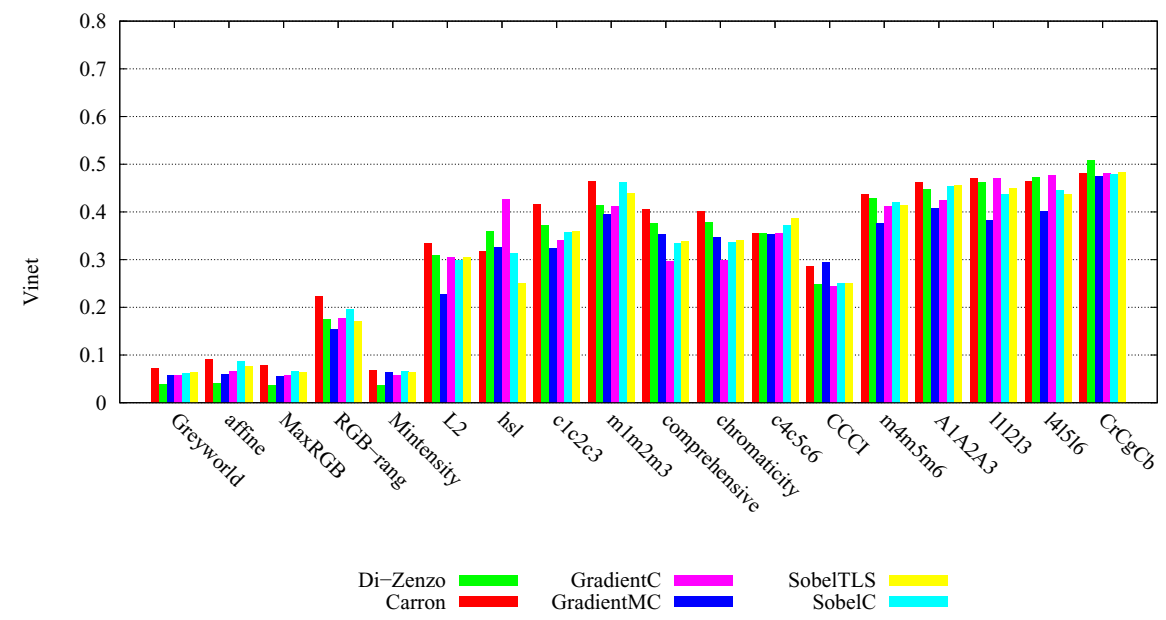

Fig. 6. Quality of the segmentation on color image (using Vinet criteria) according to the couple colorimetric invariant/gradient 


\section{Conclusion}

An image segmentation method based on watershed algorithm using appropriate couple of colorimetric invariant/gradient is presented. This segmentation method is proposed in the field of "orthophotoplan" images segmentation for roof detection. The tests performed on database with 67 roofs show that the Di-Zenzo gradient coupled to the Maximum-intensity invariant gives the best results if we consider the color information. In opposition, if we consider the gray level information, the Prewitt gradient coupled with the X component of the L2 invariant gives the best results. The proposed approach permits to conclude on the importance of the colorimetric invariant and gradient used in the segmentation step for our application. Future works concern the extraction of roof measures in order to create automatically several types of 3D model of roof.

Acknowledgements. The authors would like to thank the Volubilis program for their financial support, and the "Communauté d'agglomération Belfortaine" for the "orthophotoplan" images on which the tests have been performed.

\section{References}

1. Gevers, T., Smeulders, A.: Object Recognition based on Photometric Colour Invariants. In: Proceedings of SCIA, Lappeenranta, Finland (1997)

2. Cong, T., Khoudour, L., Achard, C., Meurie, C., Lezoray, O.: People reidentification by spectral classification of silhouettes. Signal Processing 90(8), 23622374 (2010)

3. Gouiffès, M.: Apports de la Couleur et des Modéles de Rèflexion pour l'Extraction et le Suivi de Primitives, Thése de doctorat, Université Poitiers (Décembre 2005)

4. Gevers, T., Smeulders, A.: Colour based object recognition. Pattern Recognition 32, 453-464 (1999)

5. Golland, P., Bruckstein, A.M.: Motion from color. Computer Vision and Image Understading 68(3), 346-362 (1997)

6. Schaefer, G.: How useful are colour invariants for image retrieval. In: Computational Imaging and Vision, Proc. Int. Conference on Computer Vision and Graphics, Warsaw, Poland, vol. xx. Kluwer Academic Publishers, Dordrecht (2004)

7. Hordley, D., Finlayson, G.D., Schaefer, G., Tian, G.Y.: Illuminant and device invariant colour using histogram equalization. Pattern Recognition 28, 179-190 (2005)

8. Fusiello, A., Trucco, E., Tommasini, T., Roberto, V.: Improving feature tracking with robust statistics. Pattern Analysis \& Applications 2(4), 312-320 (1999)

9. Gevers, T., Stockman, H.: Robust histogram construction from color invariants for object recognition. IEEE Transactions on Pattern Analysis and Machine Intelligence 26, 113-118 (2004)

10. Hordley, S.D., Finlayson, G.D., Schaefer, G., Tian, G.Y.: Illuminant and device invariant colour using histogram equalization. Elsevier Science, Amsterdam (2002)

11. Latecki, L.J., Rajagopal, V., Gross, A.: Image retrieval and reversible illumination normalization. In: Proc. of the IS\&T/SPIE. Internet Imaging VI, San Jose (2005)

12. Dargham, J.A.: Lip detection by the use of neural networks. Artif. Life Robotics 12, 301-306 (2008) 
13. Cousty, J.: Lignes de partage des eaux discrétes: théorie et application a la segmentation d'images cardiaques. PhD thesis, Université Marne-la-Vallé (2007)

14. Meyer, F.: Un algorithme optimal de ligne de partage des eaux. In: Dans Actes du 8éme Congrés AFCET, Lyon-Villeurbanne, France, pp. 847-859 (1991)

15. Vincent, L., Soille, P.: Watersheds in digital spaces. An efficient algorithm based on immersion simulations. IEEE Trans. Pattern Analysis and Machine Intelligence 13(6), 583-598 (1991)

16. Cousty, J.: Lignes de partage des eaux discrétes: théorie et application a la segmentation d'images cardiaques. PhD thesis, Université Marne-la-Vallé (2007)

17. Di Zenzo, R.: A note on the gradient of a multiimage. Computer Vision, Graphics and Image Processing 33, 116-125 (1986)

18. Lezoray, O., Elmoataz, A., Cardot, H., Revenu, M.: Segmentation d'images couleur: applications en microscopie cellulaire. Traitement du Signal 17, 33-45 (2007)

19. Carron, T.: Segmentation d'images couleur dans la base Teinte Luminance Saturation: approche numérique et symbolique. Thèse de doctorat, Thèse de l'Université Savoie soutenue (décembre 1995)

20. Cohen, A., Attia, D., Meurie, C., Ruichek, Y.: Une méthode de segmentation hybride par combinaison adaptative des informations texture et couleur. In: Conférence MAJESTIC, Bordeaux, France (2010)

21. Vinet, L.: Segmentation et mise en correspondance de régions de paires d'images stéréoscopiques. PhD thesis, Université Paris IX Dauphine, Juillet (1991)

22. Gevers, T., Stockman, H.: Classifying of color edges in video into shadow-geometry, highlight, or material transitions. IEEE Transactions on Multimedia 5(2), 237-243 (2003) 